\section{Dr. Khan, Dr. Hanley, and Dr. Peters reply}

\section{To the Editor:}

We thank Dr. Fung and Dr. Campisi and colleagues for their responses.

The points raised by Dr. Fung are of value and have been emphasized in the editorial recently published in The Journal ${ }^{1}$. His other comments pertaining to the need to adjudicate reported cases have also been stressed in our systematic review on bisphosphonate (BP) associated osteonecrosis of the jaw $(\mathrm{ONJ})^{2}$. The systematic review provides the incidence data in patient-years to ensure that these data are not misinterpreted. BP are of great benefit in the management of osteoporosis and other metabolic bone disorders, and if utilized appropriately according to the recently published guidelines ${ }^{3}$ are safe and effective agents. Data in the Canadian survey will shed more light on this issue, as pointed out by Dr. Fung, and are eagerly awaited.

In response to the issues presented by Dr. Campisi and colleagues:

1. We are not claiming that there is no association between low-dose BP use in the osteoporosis patient population and $\mathrm{ONJ}$, but rather that the true incidence is low enough to raise doubts that it differs from conditions occurring in the general population that can easily be classified as ONJ. It is our understanding that the cases reported to Merck were unadjudicated, and undoubtedly included dental conditions other than ONJ. The quality of published reports on BP associated ONJ have been variable and it was necessary to make decisions regarding the emphasis the various papers should be accorded. In particular, with respect to the oral BP, we felt it was premature to accept purported incidence figures. In this regard, we do not believe that cases derived from class action lawsuits, obtained by lawyers actively canvassing for clients, should be part of a scientific discussion. Physicians should be cautioned against using litigation proceedings as an indicator of the true frequency of a condition.

2. Campisi, et al are confusing our observation that many patients have been frightened into stopping their osteoporosis therapies by sensational news reports with our conservative recommendations for stopping BP use prior to invasive dental procedures. Although we have no evidence that discontinuation of $\mathrm{BP}$ prior to dental procedures offers any protection against ONJ, because of the unlikely harm in stopping the BP for several months, we do recommend interruption of BP treatment prior to invasive dental procedures. The use of "drug holidays" requires further study and has been recognized in our repor as a knowledge gap.

3. Canadian dental recommendations include regular assessments on a 6monthly basis for all Canadians. If a patient is receiving "appropriate dental care" and has "good oral hygiene," we fail to see the benefit provided by another dental examination in a patient with osteoporosis being prescribed low-dose BP therapy. It is not clear if Campisi, et al are seriously suggesting that a patient with osteoporosis should be denied treatment with our most effective medications if they have evidence of mild periodontal dis- ease. Our recommendations for the oncology patient, however, did include a thorough dental examination prior to initiating high-dose BP therapy. 4. We agree that there are multiple factors that can predispose to ONJ. However, ONJ does occur spontaneously without exposure to BP. "Lingual mandibular sequestration" occurs in healthy adults, yet resembles mild forms of BP associated ONJ. In light of these considerations, characterizing every patient who has used oral BP and also develops ONJ as an example of BP associated ONJ does not seem reasonable. The concern is that this preemptively assigns an etiologic implication to the use of BP and may lead to an obvious selection bias in studies that should be attempting to assess the contribution of the drug to the ONJ problem. There is clearly an urgent need for controlled studies.

5. Imaging technologies can help characterize $\mathrm{ONJ}$ and these will undoubtedly continue to improve, along with other methods. We agree that there is a role for radiologic investigation in ONJ, and recommended this in the assessment of oncology patients receiving high-dose intravenous BP.

The role of imaging is described in detail in our systematic review ${ }^{2}$. It is important to note, however, that the clinical presentation remains the most important element in diagnosis and the American Association of Oral and Maxillofacial Surgeons, in their 2007 position paper ${ }^{4}$, exclusively use clinical criteria to define these cases.

ALIYA KHAN, MD, FRCPC, FACP, FACE, Professor of Clinical Medicine, Divisions Endocrinology and Geriatrics, McMaster University, Hamilton, ON, Canada; DAVID A. HANLEY, MD, FRCPC, Professor, Departments of Medicine, Community Health Sciences, and Oncology, Division of Endocrinology and Metabolism, University of Calgary Health Sciences Centre, Calgary, AB, Canada; EDMUND PETERS, DDS, MSc, FRCDC, Professor, Oral Pathology and Oral Medicine, Department of Dentistry, Faculty of Medicine and Dentistry, University of Alberta, Edmonton, AB, Canada. Address reprint requests to Dr. Khan; E-mail: avkhan@aol.com

\section{REFERENCES}

1. Khan AA. Osteonecrosis of the jaw: new developments in an old disease [editorial]. J Rheumatol 2008;35:547-9.

2. Khan AA, Sándor GKB, Dore E, et al. Bisphosphonate associated osteonecrosis of the jaw [review]. J Rheumatol 2009;36: [in press].

3. Khan AA, Sándor GK, Dore E, et al. Canadian consensus practice guidelines for bisphosphonate associated osteonecrosis of the jaw. J Rheumatol 2008;35:1391-7.

4. Advisory Task Force on Bisphosphonate-Related Osteonecrosis of the Jaws. American Association of Oral and Maxillofacial Surgeons position paper on bisphosphonate-related osteonecrosis of the jaws. J Oral Maxillofac Surg 2007;65:369-76.

J Rheumatol 2009;36:2; doi:10.3899/jrheum.080808 